\title{
CATALOG OF ASTRONOMICAL POSITIONS OF SATURN'S MOONS OBTAINED BY PHOTOGRAPHIC OBSERVATIONS AT THE MAO NASU IN 1961-1991
}

\author{
O.M.Yizhakevych, V.M.Andruk, L.K.Pakuliak \\ Main Astronomical Observatory of National Academy of Sciences, \\ 27 Akad. Zabolotnogo St., 03680, Kyiv, Ukraine, izhak@mao.kiev.ua
}

\begin{abstract}
In the framework of UkrVO national project the new methods of plate digital image processing are developed. The photographic material of the UkrVO Joint Digital Archive (JDA, http://194.44.35.19/vo-mao/DB/ archivespecial.php) is used for the solution of classic astrometric problem - positional and photometric determinations of objects registered on the plates including Saturn's moons. The results of tested methods show that the positional RMS errors are better than \pm 150 mas for both coordinates and photometric ones are better than $\pm 0.20^{\mathrm{m}}$ with the Tycho- 2 catalogue as reference.
\end{abstract}

Keywords: astrometry - methods: catalogs - planets and satellites: individual: Saturn

\section{Introduction}

A decade ago we created the database of metadata of photographic astronomical plates DBGPA on the base of the MAO NASU glass archive. Later it formed the basis for the creation of Ukrainian VO Joint Digital Archive (JDA) (Vavilova et al., 2012, Vavilova et al., 2012). The efficient structure of the JDA database facilitates a quick search and selection of the desired information for the solution of specific problems such as the determination of the coordinates of celestial bodies and creation of catalogues of stars and galaxies (Kazantseva et al., 2015).

Here we present the results of the reduction of photographic observations of Saturn and its moons, made in 1961 - 1991 at the MAO NASU using 4 telescopes. In DBGPA, we found the data of almost 300 photographic observations of these objects. For further processing, we select 253 plates matching the quality criteria. These plates were scanned using Epson Expression 10000XL (EE) commercial scanner. The complete reduction of the set of plates was made using the software package specially developed and customized for this case at the MAO NASU Department of Astrometry. The catalogue of more than 1100 astrometric positions of the eight Saturn's moons (S2-S9) was obtained in the system of the Tycho-2 star catalogue. The internal accuracy of RMS reduction on the data of three telescopes DLFA, DAZ, and Z600 is about $\pm 0.03^{\prime \prime}- \pm 0.11^{\prime \prime}$, for the DWA plates the accuracy appears to be a little bit worse $\pm 015^{\prime \prime}- \pm 0.21^{\prime \prime}$. The temporal distribution of calculated positions of moons is presented in the histogram of Fig.1.
The most effective observations were those done in 1980 and 1982 with DWA and the observations conducted in 1990 with Z600 (Yizhakevych et al., 2014).

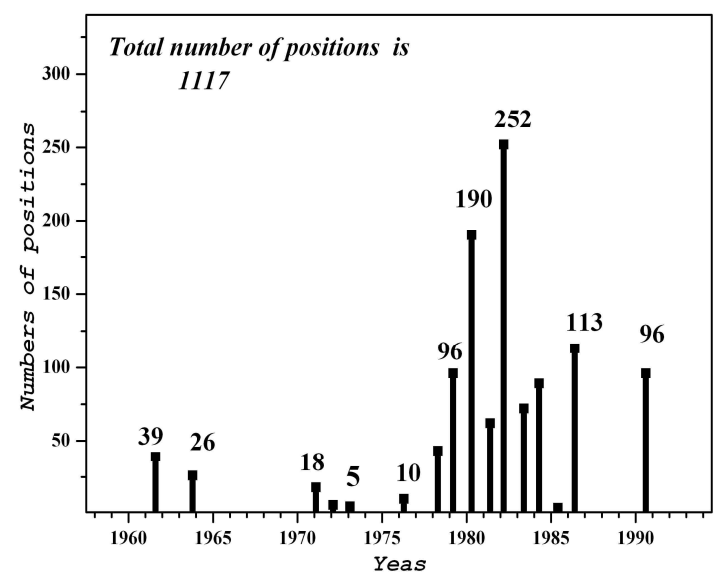

Figure 1: The distribution of the calculated positions of Saturn's moons observations using 4 telescopes of the MAO NASU.

\section{Observational data}

The first series of photographic observations (193 plates, 71 observational nights) were obtained during 1961-1988 using two astrographs: Double Long Focus Astrograph DLFA (D/F=400/5500 mm) and Double Wide Angle Astrograph DWA (D/F= 400/2000). A few short series of observations were made in field conditions: in 1986 with the Double Zeiss Astrograph DAZ (D/F= 400/3000) in Kitab observatory of the Tashkent University (Uzbekistan) (8 plates, 7 observational nights) and in 1989 (7 observational nights), 1990 (9 nights), 1991 (7 nights) with the Zeiss Reflector Z600 (F=7500 mm; mt. Maidanak, Usbekistan (54 plates). Table 1 presents the more information about the telescopes and the observational material.

The most efficient observers for 30 years who collected data for more than 10 plates each were Ledovskaya (Kulyk) I.V., Lysiakova R.F., Major S.P., Onegina A.B., Sereda E.M., Shatokhina S.V., Yizhakevych O.M.

The technique of observations of moving objects with a different brightness (from $8^{\mathrm{m}}$ to $14^{\mathrm{m}}$ ) is the exposition of multiple images of varying duration on the same plate. Obviously, the effectiveness of such observations depends 
on the mutual random placement of the Saturn's moons at the moment.

Table 2 shows the availability of Saturn's moons observations using MAO NASU telescopes. The highlighted data apply to the maximum distances between the moon and the planet (in $\mathrm{mm}$ ) on the plate depending on the telescope scale. In the other words, they concern the cases when the moon being in the opposition can be found on the plate.

Table 1. Additional information about telescopes.

\begin{tabular}{|c|c|c|c|c|}
\hline $\begin{array}{c}\text { Tele- } \\
\text { scope, } \\
\text { Tube }\end{array}$ & $\begin{array}{c}\text { Scale } \\
\text { "/mm }\end{array}$ & $\begin{array}{c}\text { Field } \\
\text { degree }\end{array}$ & $\begin{array}{c}\text { Emul- } \\
\text { sion }\end{array}$ & Years \\
\hline $\begin{array}{c}\text { DLFA, } \\
\text { I }\end{array}$ & 37.5 & $2.5 \times 2.5$ & $\begin{array}{c}\text { ORWO } \\
- \text { ZU21, } \\
\text {-ZU2 }\end{array}$ & $\begin{array}{c}1961- \\
1984\end{array}$ \\
\hline $\begin{array}{c}\text { DLFA, } \\
\text { II }\end{array}$ & 37.5 & $1.8 \times 2.5$ & $\begin{array}{c}\text { ORWO } \\
\text {-NP27 }\end{array}$ & 1982 \\
\hline $\begin{array}{c}\text { DWA, } \\
\text { I,II }\end{array}$ & 103.1 & $8.0 \times 8.0$ & $\begin{array}{c}\text { ORWO } \\
- \text { ZU21 }\end{array}$ & $\begin{array}{c}1976- \\
1988\end{array}$ \\
\hline $\begin{array}{c}\text { DAZ, } \\
\text { I,II }\end{array}$ & 68.8 & $0.5 \times 0.5$ & $\begin{array}{c}\text { ORWO } \\
\text {-ZU21 }\end{array}$ & 1986 \\
\hline $\begin{array}{c}\text { Z-600 } \\
\text { ORWO }\end{array}$ & 27.5 & $5.5 \times 5.5$ & $\begin{array}{c}\text {-ZU21, } \\
- \text { ZU2, } \\
- \text { NP27 }\end{array}$ & $\begin{array}{c}1989- \\
1991\end{array}$ \\
\hline
\end{tabular}

Table 2. The availability of the observations of Saturn's satellites (S2-S9) with telescopes of the MAO NASU.

\begin{tabular}{|c|c|c|c|c|c|}
\hline \multirow{2}{*}{$\begin{array}{l}\text { Satel- } \\
\text { lite }\end{array}$} & MgV & \multicolumn{5}{|c|}{$\begin{array}{l}\text { Distance from the Planet (mm) on the } \\
\text { plate depending on the telescope scale }\end{array}$} \\
\cline { 2 - 6 } & & $\begin{array}{c}\text { DLFA } \\
37 " / \mathrm{mm}\end{array}$ & $\begin{array}{c}\text { DWA } \\
103 " / \mathrm{mm}\end{array}$ & $\begin{array}{c}\text { DAZ } \\
68 " / \mathrm{mm}\end{array}$ & $\begin{array}{c}\text { Z600 } \\
27 " / \mathrm{m} \\
\mathrm{m}\end{array}$ \\
\hline 1 & 2 & 3 & 4 & 5 & 6 \\
\hline S2 & 11.7 & $\underline{\mathbf{1 . 1}}$ & 0.4 & 0.6 & $\underline{\mathbf{1 . 5}}$ \\
\hline S3 & 10.3 & $\underline{\mathbf{1 . 4}}$ & 0.5 & 0.7 & $\underline{\mathbf{1 . 8}}$ \\
\hline S4 & 10.4 & $\underline{\mathbf{1 . 7}}$ & 0.6 & $\underline{\mathbf{0 . 9}}$ & $\underline{\mathbf{2 . 3}}$ \\
\hline S5 & 9.7 & $\underline{\mathbf{2 . 4}}$ & $\underline{\mathbf{0 . 9}}$ & $\underline{\mathbf{1 . 3}}$ & $\underline{\mathbf{3 . 2}}$ \\
\hline S6 & 8.3 & $\underline{\mathbf{5 . 5}}$ & $\underline{\mathbf{2 . 2}}$ & $\underline{\mathbf{3 . 1}}$ & $\underline{\mathbf{7 . 5}}$ \\
\hline S7 & 14.2 & 6.7 & 2.7 & 3.7 & $\underline{\mathbf{9 . 1}}$ \\
\hline S8 & 11 & $\underline{\mathbf{1 6 . 1}}$ & $\underline{\mathbf{5 . 9}}$ & $\underline{\mathbf{8 . 9}}$ & $\underline{\mathbf{2 1 . 8}}$ \\
\hline S9 & 16.4 & 111. & 41. & 62. & 152. \\
\hline \multicolumn{5}{|c|}{ T exposure: from some seconds of time to some minutes } \\
\hline
\end{tabular}

In fact, various reasons make the chance for the satellite detection lower, for example, because of photographic irradiation, mismatched exposure duration, unfavorable mutual placement of moons, and others.

The special attention was paid to the estimation of the moments of observation. As a rule, the observer recorded the moments of cassette shutter opening and closing at the hearing using stellar chronometer or stellar clock with the further connection to the precise time signals by the «6 points» technique. Before the start of the processing, the corrections of chronometers and stellar clocks were newly qualified and the UT moments of the middle of the exposition were also redefined for each image in contrast to DBGPA data, where the moments of observation have the different format of presentation. They are the start moments of the exposure and its duration.

Search files for the identification of the moons were prepared due to the IMCCE (Paris) ephemeris (http://www. imcce.fr/hosted_sites/aimirror/nssephe.php).

\section{Reduction}

The algorithm of calculation of the positions by photographic observations is as follows: the first step is the determination of the rectangular coordinates $\boldsymbol{x}, \boldsymbol{y}$ of objects in the plate coordinate system; on the second step, the objects are identified in the reference star catalogue, and finally the positions in the equatorial coordinate system $\boldsymbol{\alpha}, \boldsymbol{\delta}$ are derived.

The connection between two coordinate systems $\boldsymbol{x}, \boldsymbol{y}$ and $\boldsymbol{\alpha}, \boldsymbol{\delta}$ is determined by the infinite power series, whose members depend on many factors. Among them are the quality of the telescope optics, the field of view of the telescope, the tilt of the plate to the focal plane of the optical system, the reference system used, the number of reference stars on the plate and other factors. The calculations using the formulae of the linking are possible only after the application of restrictions on the number of members of the power series in the result of the evaluation of their relevance on the basis of the adopted model of aberrations. By varying this number, we can get results with the highest level of accuracy, leaving only the significant members of the series (Andruk et al., 2005; Andruk et al., 2014). The scanning of plates was made using Epson Expression 10000XL scanner at 16-bit gray levels and a resolution of 1200 dpi. (Golovnya et al., 2010). Previously, the detailed study of the device, namely the additional errors, connected with the scanner mechanics behavior, has showed its applicability to the solution of problems of ground-based astronomy (Protsyuk et al., 2014).

The software package, specially developed and customized for the case in the LINUX-MIDAS-ROMAFOT environment (Andruk et al., 2007; Andruk et al., 2010), allowed to make a reduction of $\mathbf{2 5 3}$ scans with Saturn's moons images. In fact, the amount of processed material appeared to be approximately three times larger because of multiple images on the each plate. It requires the additional efforts to improve the software algorithm for splitting the digitized image into separate files according to the number of exposures on the plate.

Initially, the software was developed for the processing of the astronegatives of the FON project (Yatsenko et al., 2011). Its plates have large fields of view and are obtained with two expositions: long (16-20 min.) and short (30-60 sec) ones. This allows selecting a few hundred reference stars from the Tycho- 2 catalogue homogeneously distributed across the plate field. The more reference stars are on the plate, the more accurate solution for the relation of two coordinate systems could be obtained. In our case, the number of exposures exceeds two.

The processing of the DWA $\left(8^{\circ} \times 8^{\circ}\right)$ and DAZ $\left(5^{\circ} \times 5^{\circ}\right)$ plates was made using the polynomial of the sixth degree. In addition to them in the observations of Saturn's moons another two telescopes were involved having different 
scales of images and star fields (Table 1$)$. So, the reduction of DLFA plates $\left(2^{\circ} \times 2^{\circ}\right)$ needs the polynomial of the third degree, and the linear model was applied to the reduction of $\mathrm{Z} 600\left(0.5^{\circ} \times 0.5^{\circ}\right)$ plates.

The first step of the reduction includes the full analysis of the image for detecting the blemishes and damages of the emulsion on the plate as well as their removal. The elimination of scanner errors is done on the same step too.

The next step was the full reduction of the selected plates. On this step, the plates with failed observations were found and excluded from the processing. These include plates with aiming errors (plates of Z600, obtained in 1991), improper exposition duration when observing faint objects (plates with Phoebe - S9, 16.4 ; plates of Z600, obtained in 1989), errors in the recording of times and in the determination of chronometer corrections, etc.

Finally, we obtained the catalogue of 1100 astro-metric positions of eight Saturn's moons (S2-S9) using 193 photographic plates exposed on four telescopes during 87 productive observational nights.

The comparison of observed positions with the theory of moons' motion DE431 was made using the ephemeris of IMCCE, France, and the residuals $(\mathrm{O}-\mathrm{C})_{\alpha}$ and $(\mathrm{O}-\mathrm{C})_{\delta}$, as well as their standard deviations $\sigma$, were obtained according to (1):

$$
\sigma=\sqrt{\Sigma\left((o-c)_{i}-\overline{(o-c)}\right)^{2} /(n-1)} .
$$

The results of the comparison are given in Table 3. The internal accuracy of the astrometric reduction into the Tycho-2 reference system lies within \pm 0.03 " $- \pm 0.11^{\prime \prime}$ for the three telescopes DLFA, DAZ, Z600. For the DWA, the internal accuracy is worse, and it is equal $\pm 015^{\prime \prime}- \pm 0.21$ ".

Figures 2, 3, 4, 5 show the scattering of $(\mathrm{O}-\mathrm{C})$ deviations from their average value for S6 satellite as the most efficient object for all four telescopes. The comparison between these four histograms demonstrates that the scattering of $\mathrm{O}-\mathrm{C}$ from their average value lies in the limits of 1 " and is random for DLFA, DAZ and Z600. In the case of DWA, this scattering is much larger and lies within \pm 2 ". The dispersion analysis for the Fisher test shows that it may indicate the presence of an undetected source of residual systematic errors.

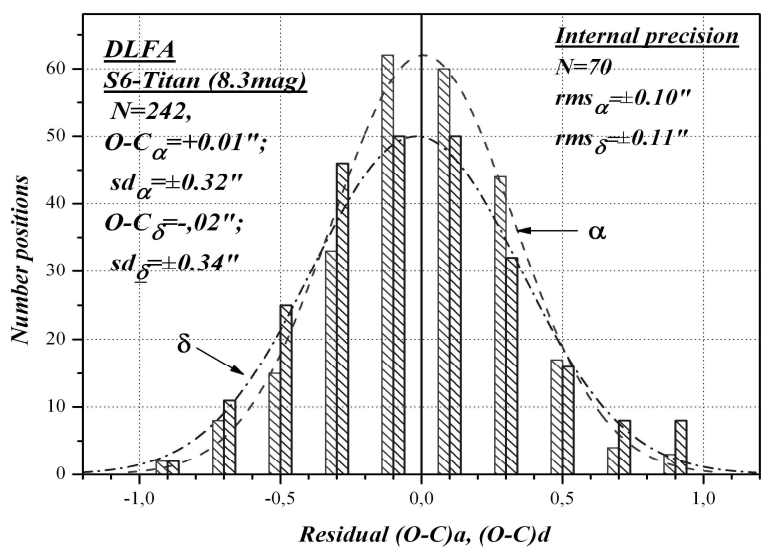

Figure 2: Residuals (O-C); DLFA
Table 3. Statistic parameters of the results of the astrometric solution for 7 satellites and 4 telescopes.

\begin{tabular}{|c|c|c|c|c|c|}
\hline \multirow{2}{*}{ Obj } & \multicolumn{5}{|c|}{ DLFA (1961-1984 yrs) } \\
\hline & $\mathrm{N}$ & $\mathrm{O}-\mathrm{C}_{\alpha} \prime$ & $\sigma_{\alpha}^{\prime \prime}$ & $\mathrm{O}-\mathrm{C}_{\delta} \prime$ & $\sigma_{\delta}^{\prime \prime}$ \\
\hline $\mathrm{S} 2$ & 9 & +.402 & 0.32 & +.184 & 0.17 \\
\hline S3 & 71 & -.094 & 0.38 & +.085 & 0.46 \\
\hline $\mathrm{S} 4$ & 118 & -.015 & 0.33 & +.067 & 0.39 \\
\hline S5 & 186 & +.071 & 0.34 & +.018 & 0.35 \\
\hline S6 & 248 & +.005 & 0.32 & -.018 & 0.37 \\
\hline S8 & 212 & -.019 & 0.36 & +.019 & 0.37 \\
\hline$\Sigma$ & 844 & +.007 & \pm .34 & +.022 & \pm .37 \\
\hline \multirow{2}{*}{ Obj } & \multicolumn{5}{|c|}{ DWA (1978-1986 yrs) } \\
\hline & $\mathrm{N}$ & $\mathrm{O}-\mathrm{C}_{\alpha}{ }^{\prime \prime}$ & $\sigma_{\alpha}^{\prime \prime}$ & $\mathrm{O}-\mathrm{C}_{\delta}{ }^{\prime \prime}$ & $\sigma_{\delta}^{\prime \prime}$ \\
\hline S5 & 5 & -.744 & 0.85 & -.214 & 1.11 \\
\hline S6 & 55 & +.209 & 0.60 & +.109 & 0.64 \\
\hline S8 & 36 & -.744 & 0.85 & -.214 & 1.11 \\
\hline$\Sigma$ & 96 & -.198 & \pm .72 & -.004 & \pm .87 \\
\hline \multirow{2}{*}{ Obj } & \multicolumn{5}{|c|}{ Z600 (1990) } \\
\hline & $\mathrm{N}$ & $\mathrm{O}-\mathrm{C}_{\alpha}{ }^{\prime \prime}$ & $\sigma_{\alpha}^{\prime \prime}$ & $\mathrm{O}-\mathrm{C}_{\delta}{ }^{\prime \prime}$ & $\sigma_{\delta}^{\prime \prime}$ \\
\hline S3 & 4 & +.166 & 0.25 & +.041 & 0.30 \\
\hline $\mathrm{S} 4$ & 11 & -.160 & 0.30 & +.089 & 0.24 \\
\hline S5 & 12 & -.252 & 0.46 & +.183 & 0.26 \\
\hline S6 & 35 & -.032 & 0.43 & -.219 & 0.30 \\
\hline S7 & 5 & -.252 & 0.51 & +.099 & 0.39 \\
\hline S8 & 29 & +.313 & 0.41 & +.016 & 0.34 \\
\hline S9 & 1 & +.439 & & -.268 & \\
\hline $\boldsymbol{\Sigma}$ & 97 & +.027 & \pm .41 & -.035 & \pm .31 \\
\hline \multirow{2}{*}{ Obj } & \multicolumn{5}{|c|}{ DAZ (1986) } \\
\hline & $\mathrm{N}$ & $\mathrm{O}-\mathrm{C}_{\alpha}{ }^{\prime \prime}$ & $\sigma_{\alpha}^{\prime \prime}$ & $\mathrm{O}-\mathrm{C}_{\delta}{ }^{\prime \prime}$ & $\sigma_{\delta}^{\prime \prime}$ \\
\hline S4 & 4 & -.451 & 0.27 & +.096 & 0.15 \\
\hline S5 & 18 & -.093 & 0.47 & +.070 & 0.29 \\
\hline S6 & 29 & -.052 & 0.34 & +.040 & 0.27 \\
\hline S8 & 29 & -.012 & 0.31 & -.012 & 0.31 \\
\hline $\boldsymbol{\Sigma}$ & 80 & -.055 & \pm .33 & +.025 & \pm .26 \\
\hline
\end{tabular}

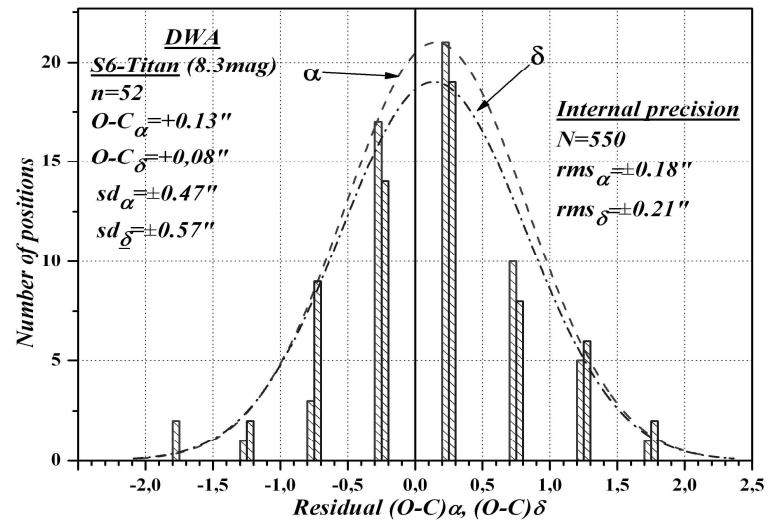

Figure 3: Residuals (O-C); DWA. 


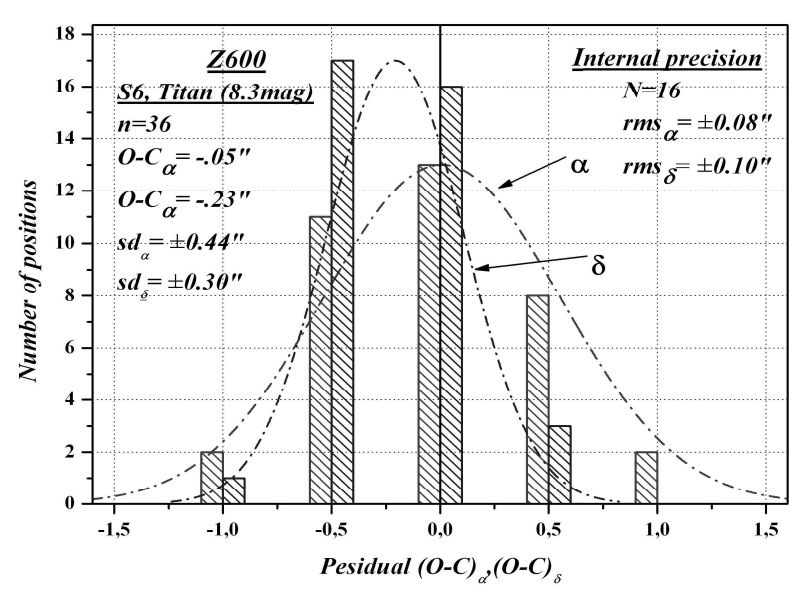

Figure 4: Residuals (O-C); Z600.

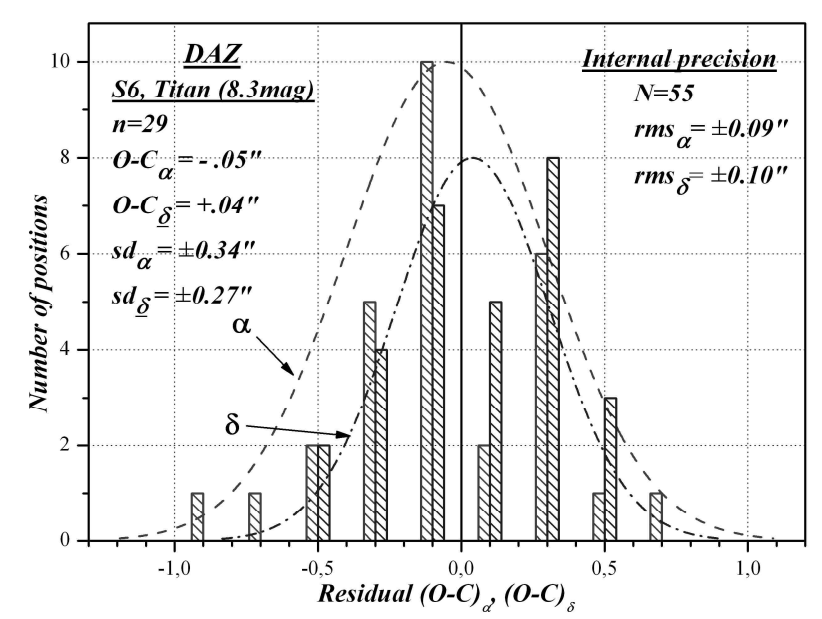

Figure 5. Residuals (O-C); DAZ.

\section{Conclusion}

The catalogue of 1100 astrometric positions of eight Saturn's moons S2-S9 was obtained based on the reduction of four independent series of observations from different telescopes. For the moment, we have processed 193 plates from the set of 253 plates found in the database of UkrVO JDA.

The internal accuracy of the reduction for three telescopes with the scales from 28 to $68 \mathrm{arcsec} / \mathrm{mm}$ lies within $\pm 0.03^{\prime \prime}- \pm 0.11^{\prime \prime}$. The fourth instrument with the scale $103 \mathrm{arcsec} / \mathrm{mm}$ gives the accuracy in the interval $\pm 015^{\prime \prime}-$ $\pm 0.21^{\prime \prime}$ and the larger dispersion of $\mathrm{O}-\mathrm{C}$.

For three telescopes DLFA, DAZ, Z600 standard deviations $\sigma$ are in the range from $\pm 0.25^{\prime \prime}$ to $\pm 0.37^{\prime \prime}$ for both coordinates, while those for DWA exceed the above values of 1.5-2 times.

The dispersion analysis for the Fisher test shows that the larger dispersion of O-C for DWA may indicate the presence of an undetected source of residual systematic errors. In order to identify the cause of these errors, we assume to perform the additional analysis of the reduction of DWA observations.

The problem of the reduction of Z600 plates exists. Most of them were eliminated because of insufficient results of the preliminary digital analysis of images for defects and damages of emulsions. The considerable number of moons' images close to the planet was not found on the scans of those plates using the digital procedure in contrast to the previous reductions based on the classic technique of measurements (Izhakevich, 1991; Yizhakevych, 1991; Yizhakevych, 1994, Kaltygina, 1992; Pakuliak, 2012; Yizhakevych, 2012). These points to the possible limitations of the processing algorithm, which could misidentify the few pixel images of faint moons as the "dirt" on the emulsion. This requires the further improvement of the software for the processing of digital images of plates with the Solar System bodies.

Acknowledgements. This work was partially supported by the Ukrainian Astronomical Association.

\section{References}

Andruk V.M. et al.: 2005, Kinem. Phys. Cel. Bodies, 21, N5, 396.

Andruk V.M. et al.: 2007, J. Phys. Studies, 11, N3, 329.

Andruk V.M. et al.: 2010, Kinem. Phys. Cel. Bodies, 26, N3, 75 .

Andruk V.M. et al.: 2014, Kinem. Phys. Cel. Bodies, 27, N1, 53.

Golovnya V.V. et al.: 2010, J. Phys. Studies, 14, N2, 2902.

Izhakevich E.M.: 1991, Scientific paper deposited in Allrussian institute of scientific and technical information, No. 4553-B91, 1991PDRII4553 I.

Kaltygina S.V. et al.: 1992, Scientific paper deposited in All-russian institute of scientific and technical information, No. 1044-Uk92, 5.

Kazantseva L.V. et al.: 2015, Kinem. Phys. Cel. Bodies, 31, N1, 58.

Pakuliak L. et al.: 2012, in Proc. of the Conf. NAROOGAIA "A new reduction of old observations...", June 20-22, 2012, France, Paris, p. 161.

Protsyuk Yu.I. et al: 2014, Odessa Astron. Publ., 27/1, 59.

Vavilova I.B. et al.: 2012a, Kinem. Phys. Cel. Bodies, 28 N2, 85 .

Vavilova I.B. et al.: 2012b, Baltic Asronomy, 21, N3, 356.

Yatsenko, A.I. et al.: 2011, Kinem. Phys. Cel. Bodies, 27, N5, 249.

Yizhakevych O.M. et al.: 1991, Kinem. Phys. Cel. Bodies, 7, №2, 98.

Yizhakevych O.M. et al.: 1994, Kinem. Phys. Cel. Bodies, 10, №1, 88 .

Yizhakevych Ye. et al.: 2012, in Proc. of the Conf. NAROO-GAIA "A new reduction of old observations...", June 20-22, 2012, France, Paris, p. 153.

Yizhakevych O. et.al.: 2014, Odessa Astron. Publ., 27/1, 67. 